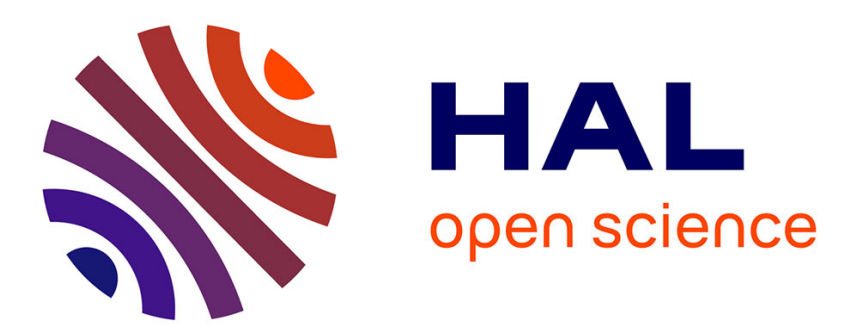

\title{
Equilibria of a Class of Transport Equations Arising in Congestion Control
}

\author{
François Baccelli, Ki Baek Kim, David R. Mcdonald
}

\section{To cite this version:}

François Baccelli, Ki Baek Kim, David R. Mcdonald. Equilibria of a Class of Transport Equations Arising in Congestion Control. [Research Report] RR-5653, INRIA. 2005, pp.19. inria-00070357

\section{HAL Id: inria-00070357 https://hal.inria.fr/inria-00070357}

Submitted on 19 May 2006

HAL is a multi-disciplinary open access archive for the deposit and dissemination of scientific research documents, whether they are published or not. The documents may come from teaching and research institutions in France or abroad, or from public or private research centers.
L'archive ouverte pluridisciplinaire HAL, est destinée au dépôt et à la diffusion de documents scientifiques de niveau recherche, publiés ou non, émanant des établissements d'enseignement et de recherche français ou étrangers, des laboratoires publics ou privés. 


\title{
Equilibria of a Class of Transport Equations Arising in Congestion Control
}

\author{
Francois Baccelli — Ki Baek Kim — David R. McDonald
}

$\mathbf{N}^{\circ} 5653$

Août 2005

Thème COM

\section{apport}

derecherche 



\title{
Equilibria of a Class of Transport Equations Arising in Congestion Control
}

\author{
Francois Baccelli * , Ki Baek Kim ${ }^{\dagger}$, David R. McDonald ${ }^{\ddagger}$ \\ Thème COM — Systèmes communicants \\ Projet TREC
}

Rapport de recherche $\mathrm{n}^{\circ} 5653$ - Août 2005 - 19 pages

\begin{abstract}
This paper studies a class of transport equations arising from stochastic models in congestion control. This class contains two cases of loss point process models: the rate-independent Poisson case where the packet loss rate is independent of the throughput of the flow and the rate-dependent case where the point process of losses has an intensity which is a function of the instantaneous rate. This class of equations covers both the case of persistent and of non-persistent flows. We give a direct proof of the fact that there is a unique density solving the associated differential equation and we provide a closed form expression for this density and for its mean value.
\end{abstract}

Key-words: Flow control, congestion avoidance, TCP, HTTP, AIMD, stochastic process, Poisson point process, stochastic intensity, stationary density, change of measure.

This work was funded in part by the Alcatel-INRIA OSC 'End-to-End Performance Evaluation of Packet Networks', by the EuroNGI European Network of Excellence, and by the Canadian NSERC grant A4551.

* \{Francois.Baccelli@ens.fr $\}$, INRIA-ENS, Département d'Informatique, Ecole Normale Supérieure, 45, rue d'Ulm F-75230 Paris cedex 05, France.

$\dagger\{k k b @$ di.ens.fr $\}$, INRIA-ENS, Département d'Informatique, Ecole Normale Supérieure, 45, rue d'Ulm F-75230 Paris cedex 05, France.

$\ddagger$ ddmdsg@uottawa.ca\}, Department of Mathematics and Statistics, University of Ottawa, 585 King Edward Avenue, Ottawa, K1N6N5 Ontario Canada. 


\section{Sur les Solutions Stationnaires d'une Classe d'Équations de Transport Utilisées en Contrôle de Congestion}

Résumé : Nous étudions dans cet article une classe d'équations de transport qui apparaissent dans des modèles stochastiques de mécanismes de contrôle de congestion. Cette classe couvre deux modèles de processus ponctuels de pertes: le cas où les pertes forment un processus de Poisson, et celui où ce processus ponctuel a une intensité qui est une fonction du débit instantané du flot. Elle couvre aussi le cas des flots persistants comme celui des non-persistants. Pour cette classe, nous donnons une preuve directe de l'unicité des densités solutions de l'équation différentielle décrivant l'état stationnaire, ainsi qu'une expression explicite pour cette densité et pour sa valeur moyenne.

Mots-clés : Contrôle de flot, évitement de congestion, TCP, HTTP, AIMD, processus stochastique, processus ponctuel de Poisson, intensité stochastique, densité stationnaire, changement de mesure. 


\section{Introduction}

The aim of the present paper is to give a comprehensive mathematical treatment of a class of differential equations that characterize the steady state of some transport partial differential equations (PDEs) associated with the dynamics of TCP flows. We establish a generic equation that contains most features considered in the literature: persistent and non persistent flows, rate dependent and rate independent losses, as well as the case with slow start and timeouts.

The following ordinary differential equation (ODE) was considered in [3]

$$
\frac{d f(z)}{d z}=\beta(4 z f(2 z)-z f(z)), \quad z \geq 0,
$$

with $\beta>0$. This ODE represents the throughput density of a TCP connection in statistical equilibrium when a router drops its packets with constant probability $\beta$. The rate at which its packets are dropped is $\beta$ times the throughput so we call (1) rate-dependent. By the congestion control mechanism of TCP [2] this is also the rate at which the throughput is cut in half. The function $f$ represents the density of transmission rates in statistical equilibrium.

The intuition is as follows. The proportion of the throughputs in an interval $[z, z+d z]$ is approximately $f(x) d x$. These suffer a loss at a rate $\beta z$ and hence the rate at which mass leaves the interval $[z, z+d z]$ is $\beta z f(z) d z$ approximately. Mass enters this interval because of losses among throughputs in the interval $[2 z, 2(z+d z)]$ at rate $\beta 2 z f(2 z) \cdot 2 d z$ approximately. Mass also enters and leaves the interval because of the linear increase in the transmission rates and the rate of change of the mass in $[z, z+d z]$ is approximately $\frac{d f(z)}{d z} d z$. Equation (1) is the corresponding balance equation.

These equations also come up in the equilibrium distribution of the weights of a population of cells since the weights grow linearly until mitosis cuts the weight in half (see [1]).

The following solution of (1) was obtained by inspection in [3] and using Mellin transforms in [5]:

$$
f(z)=2 \phi \sqrt{\beta} \sum_{n \geq 0} a_{n} e^{-\left(\frac{\beta}{2} 4^{n}\right) z^{2}}
$$

$\mathrm{RR} \mathrm{n}^{\circ} 5653$ 
with $\phi=\left(\sqrt{2 \pi} \prod_{k \geq 1}\left(1-2^{-2 k+1}\right)\right)^{-1}$ and with $a_{0}=1$ and $a_{n}=(-1)^{n} \prod_{k=1}^{n} \frac{4}{\left(4^{k}-1\right)}$.

The associated transient equation is the following non-local transport PDE:

$$
\alpha \frac{\partial}{\partial t} f(t, z)+\frac{\partial}{\partial z} f(t, z)=\beta(4 z f(t, 2 z)-z f(t, z)), \quad z, t \geq 0 .
$$

This PDE has been generalized in several directions. In particular [6] has considered this equation with $4 z f(t, 2 z)-z f(t, z)$ replaced by $2 b(2 z) f(t, 2 z)-$ $b(z) f(t, z)$ where $b(z)$ is a general function. The existence of a solution as well as the exponential rate of convergence of the solution to the associated equilibrium ODE are shown. References to related work on such equations (which are often referred to as fragmentation equations) are given.

In the rate-independent case of [5], the ODE is

$$
\frac{d f(z)}{d z}=\beta(2 f(2 z)-f(z)), \quad z \geq 0
$$

with $\beta>0$ and its solution (obtained by Mellin transforms in Lemma 2 [5]) is

$$
f(z)=\phi \beta \sum_{n \geq 0} a_{n} e^{-\left(\beta 2^{n}\right) z}
$$

with $\phi=\left(\prod_{k \geq 1}\left(1-2^{-k}\right)\right)^{-1}$ and with $a_{n}=(-1)^{n} \prod_{k=1}^{n} \frac{2}{\left(2^{k}-1\right)}$ and $a_{0}=1$.

In the rate-dependent case, the non-persistent equation introduced in [4] is

$$
\frac{d f(z)}{d z}=\int_{0}^{\infty} x f(x) d x \delta A(z)-\mu z f(z)+\beta z(4 f(2 z)-f(z))
$$

where $\delta \geq 0, A$ is a probability density function, $\mu \geq 0$ and $\beta>0$. Here, the constant $\mu$ measures exogenous losses which cause the transmission rate to be instantaneously cut to zero, like timeouts in the TCP context or transmission completions in the HTTP context. The constant $\delta$ measures the rate at which transmission rates instantaneously increase from 0 , where the increase has density $A$. This is a simplified representation of the slow-start mechanism after a timeout or a transmission completion (see [4]). The case with $\delta=0$ is 
that where there is no slow start. [4] provides the density solution when $\delta=0$ and the mean value for all $\delta \geq 0$ using Mellin transforms.

In the present paper, we consider the more general non-persistent equation which allows more general rate-dependences

$$
\frac{d f(z)}{d z}=\delta T(f) A(z)-\mu z^{\gamma-1} f(z)+\beta z^{\gamma-1}\left(\rho^{\gamma} f(\rho z)-f(z)\right), \quad z \geq 0,
$$

where $\delta \geq 0, T(f)=\int_{0}^{\infty} z^{\gamma-1} f(z) d z, A$ is a probability density function with $\int_{0}^{\infty} A(z) d z=1, \mu \geq 0, \gamma \geq 1, \beta \geq 0, \rho>1$, and $\mu+\beta>0$. The associated transport PDE is defined in the same way.

Throughout the rest of this paper, we look for a solution $f(z)$ of $(3)$ which is a density such that $T(f)$ is finite. We give a direct proof that there is a unique density solving (3) and we give closed form expressions for this density and its mean value.

Integrating (3) from zero to infinity gives

$$
f(0)=(\mu-\delta) T(f) \geq 0 \quad \text { or } \quad \mu \geq \delta
$$

(since there exists a subsequence $z_{k} \rightarrow \infty$ such that $\lim _{z_{n} \rightarrow \infty} f\left(z_{n}\right)=0$ since $f$ is integrable).

The special case with $\delta=\mu=0$, namely

$$
\frac{d f(z)}{d z}=\beta z^{\gamma-1}\left(\rho^{\gamma} f(\rho z)-f(z)\right), \quad z \geq 0,
$$

will be called the persistent equation. It describes a persistent source in congestion avoidance without slow-start and where the rate dependence is proportional to $z^{\gamma-1}$. The persistent equation includes (1) and (2) as special cases.

In the next section, we summarize the two main results of this paper. A detailed proof is given in the following two sections. Finally, we study the associated PDE.

\section{Main Results}

Let $\Gamma(t)=\int_{0}^{\infty} x^{t-1} e^{-x} d x$ denote Euler's Gamma function. The following theorem summarizes our results on the persistent ODE (4):

$\mathrm{RR} \mathrm{n}^{\circ} 5653$ 
Theorem 1 If $\gamma \geq 1, \rho>1, \beta>0$, the unique density solution to (4) is

$$
f(z)=\gamma\left(\frac{\beta}{\gamma}\right)^{1 / \gamma} \frac{1}{\Gamma(1 / \gamma)} \prod_{k \geq 0} \frac{1}{\left(1-\theta^{-k-1 / \gamma}\right)} \sum_{n \geq 0} b_{n} e^{-\frac{\beta}{\gamma} \theta^{n} z^{\gamma}},
$$

where $\theta=\rho^{\gamma}, b_{0}=1$ and $b_{n}=(-1)^{n} \prod_{k=1}^{n} \frac{\theta}{\left(\theta^{k}-1\right)}$.

The $b_{n}$ 's are the coefficients of the expansion

$$
\prod_{k \geq 0}\left(1-\theta^{-k} x\right)=\sum_{n \geq 0} b_{n} x^{n} .
$$

The mean value of this density is

$$
\int_{0}^{\infty} z f(z) d z=\left(\frac{\beta}{\gamma}\right)^{-1 / \gamma} \frac{\Gamma(2 / \gamma) \prod_{k \geq 0}\left(1-\theta^{-k-2 / \gamma}\right)}{\Gamma(1 / \gamma) \prod_{k \geq 0}\left(1-\theta^{-k-1 / \gamma}\right)} .
$$

The following theorem summarizes our results on the non-persistent ODE (3):

Theorem 2 Let $\gamma \geq 1, \rho>1, \mu \geq \delta \geq 0, \beta \geq 0, \mu+\beta>0$ and $A$ be $a$ density such that

$$
\int_{0}^{\infty} A(z) e^{\frac{(\mu+\beta)}{\gamma} z^{\gamma}} d z<\infty
$$

Then the unique density solution to (3) is the function

$$
f(z)=\frac{1}{C \gamma} \sum_{n \geq 0}\left(\frac{\beta}{\mu+\beta}\right)^{n} b_{n} d_{n}(z) e^{-\left(\frac{\beta+\mu}{\gamma}\right) \theta^{n} z^{\gamma}}
$$

with

$$
\begin{aligned}
d_{n}(z) & =\sum_{m \geq 0} c_{m}\left(\delta \int_{0}^{\theta^{\frac{(n+m)}{\gamma} z}} \bar{A}_{m}(x) d x+(\mu-\delta)\right) \\
c_{m} & =\left(\frac{\beta}{\mu+\beta}\right)^{m} \prod_{i=1}^{m} \frac{1}{1-\theta^{-i}} \quad \text { and } \\
\bar{A}_{m}(x) & =A(x) e^{\frac{\mu+\beta}{\gamma} \theta^{-m} x^{\gamma}} .
\end{aligned}
$$


The constant $C$ which normalizes (8) to be a density is

$$
\begin{aligned}
C= & \frac{1}{\gamma^{2}} \prod_{k \geq 0}\left(1-\frac{\beta}{\mu+\beta} \theta^{-k-1 / \gamma}\right)\left(\sum_{m \geq 0} c_{m}\right. \\
& \left.\left(\delta \int_{0}^{\infty} \bar{A}_{m}(x) \int_{x^{\gamma} / \theta^{m}}^{\infty} B_{1}(u) d u d x+(\mu-\delta) D(1)\right)\right)
\end{aligned}
$$

with

$$
\begin{aligned}
B_{l}(u) & =\frac{e^{-\frac{(\mu+\beta)}{\gamma} u}}{u^{1-l / \gamma}} \\
D(l) & =\left(\frac{\mu+\beta}{\gamma}\right)^{-l / \gamma} \Gamma\left(\frac{l}{\gamma}\right) .
\end{aligned}
$$

The mean of $f(z)$ is

$$
\begin{aligned}
& \int_{0}^{\infty} z f(z) d z=\frac{\prod_{k \geq 0}\left(1-\theta^{-k-2 / \gamma} \frac{\beta}{\beta+\mu}\right)}{\prod_{k \geq 0}\left(1-\theta^{-k-1 / \gamma} \frac{\beta}{\beta+\mu}\right)} \\
& \sum_{m \geq 0} c_{m}\left(\delta \int_{0}^{\infty} \bar{A}_{m}(x) \int_{x^{\gamma} / \theta^{m}}^{\infty} B_{2}(u) d u d x+(\mu-\delta) D(2)\right) \\
& \sum_{m \geq 0} c_{m}\left(\delta \int_{0}^{\infty} \bar{A}_{m}(x) \int_{x^{\gamma} / \theta^{m}}^{\infty} B_{1}(u) d u d x+(\mu-\delta) D(1)\right)
\end{aligned}
$$

As we shall see later, for both the persistent and the non-persistent case, the solution of the rate-dependent case can actually be built from that of the rate-independent case by a mere change of measure. It is why we start with the latter case below.

\section{The Rate-Independent Case $(\gamma=1)$}

In the rate-independent case, (3) is given by

$$
\frac{d f(z)}{d z}=\delta A(z)-\mu f(z)+\beta(\theta f(\theta z)-f(z)), \quad z \geq 0
$$

$\mathrm{RR} \mathrm{n}^{\circ} 5653$ 
where $\theta>1$ (since we look for a density, $T(f)=\int_{0}^{\infty} f(z) d z=1$ ).

Multiplying (9) by $\exp (-s z)$ and integrating from zero to $\infty$ gives the following expression for the Laplace transform $\widehat{f}(s)$ :

$$
s \widehat{f}(s)-f(0)=\delta \widehat{A}(s)-\mu \widehat{f}(s)+\beta\left(\widehat{f}\left(\frac{s}{\theta}\right)-\widehat{f}(s)\right)
$$

that is

$$
\widehat{f}(s)=\frac{f(0)+\delta \widehat{A}(s)}{\mu+\beta+s}+\frac{\beta}{\mu+\beta+s} \widehat{f}\left(\frac{s}{\theta}\right)
$$

where $\widehat{A}$ is the Laplace transform of the density $A$. By iteration it follows that

$$
\begin{aligned}
\widehat{f}(s)= & \sum_{n=0}^{N} \frac{\beta^{n}}{\prod_{k=0}^{n}\left(\mu+\beta+s / \theta^{k}\right)} \cdot\left(f(0)+\delta \widehat{A}\left(\frac{s}{\theta^{n}}\right)\right) \\
& +\prod_{n=0}^{N}\left(\frac{\beta}{\mu+\beta+s / \theta^{k}}\right) \widehat{f}\left(\frac{s}{\theta^{N+1}}\right) .
\end{aligned}
$$

\subsection{The persistent case $(\mu=\delta=0)$}

In the persistent case, letting $N$ tend to infinity, we get

$$
\begin{aligned}
\widehat{f}(s) & =\prod_{k \geq 0} \frac{\beta}{\beta+s / \theta^{k}} \cdot \widehat{f}(0) \\
& =\prod_{k \geq 0} \frac{\beta}{\beta+s / \theta^{k}}
\end{aligned}
$$

with $\widehat{f}(0)=\int_{0}^{\infty} f(z) d z=1$.

We remark that $\widehat{f}$ is the Laplace transform of a sum of independent geometric random variables $E_{k}$ with parameter $\beta \theta^{k}$. Note that the sum of the means $\sum_{k \geq 0} \theta^{-k} / \beta<\infty$ so this random variable exists and has a positive density.

We thus have a solution for (2) with $\theta=2$. The ODE is

$$
\frac{d f(z)}{d z}=\beta(\theta f(\theta z)-f(z)), \quad z \geq 0 .
$$


The solution can also be found by the Mellin transform as in [5]:

$$
f(z)=\psi \beta \sum_{n \geq 0} b_{n} e^{-\left(\beta \theta^{n}\right) z}
$$

with $\psi=\left(\prod_{k \geq 1}\left(1-\theta^{-k}\right)\right)^{-1}$.

We can check this inversion by calculating the Laplace transform of (12):

$$
\psi \beta \sum_{n \geq 0} b_{n} \int_{0}^{\infty} e^{-u z} e^{-\left(\beta \theta^{n}\right) z} d z=\psi \beta \sum_{n \geq 0} \frac{b_{n}}{u+\beta \theta^{n}}
$$

The residue of the pole $-\beta \theta^{n}$ is $\psi \beta b_{n}$. The residue of $\widehat{f}$ given by (10) at the pole $-\beta \theta^{n}$ is

$$
\begin{aligned}
& \beta \theta^{n} \prod_{k=0}^{n-1}\left(\frac{\beta \theta^{k}}{\beta \theta^{k}-\beta \theta^{n}}\right) \prod_{k \geq n+1}\left(\frac{\beta \theta^{k}}{\beta \theta^{k}-\beta \theta^{n}}\right) \\
& =\beta \theta^{n} \prod_{k=0}^{n-1}\left(\frac{1}{1-\theta^{n-k}}\right) \prod_{k \geq n+1}\left(\frac{\theta^{k-n}}{\theta^{k-n}-1}\right) \\
& =\beta \prod_{k=1}^{n}\left(\frac{\theta}{1-\theta^{k}}\right) \prod_{k \geq 1}\left(\frac{\theta^{k}}{\theta^{k}-1}\right) \\
& =\beta \cdot b_{n} \cdot \psi .
\end{aligned}
$$

Thus we see the Laplace transform of $f$ is indeed (10).

\subsection{The non-persistent case $(\mu>\delta \geq 0)$}

Letting $N \rightarrow \infty$ we get

$$
\begin{aligned}
\widehat{f}(s) & =\sum_{n \geq 0} \frac{\beta^{n}}{\prod_{k=0}^{n}\left(\mu+\beta+s / \theta^{k}\right)}\left(f(0)+\delta \widehat{A}\left(\frac{s}{\theta^{n}}\right)\right) \\
& =\sum_{n \geq 0}\left(1-\frac{\delta}{\mu}+\frac{\delta}{\mu} \widehat{A}\left(\frac{s}{\theta^{n}}\right)\right)\left(1-\frac{\beta}{\mu+\beta}\right)\left(\frac{\beta}{\mu+\beta}\right)^{n} \prod_{k=0}^{n} \frac{\mu+\beta}{\mu+\beta+s / \theta^{k}}
\end{aligned}
$$

$\mathrm{RR} \mathrm{n}^{\circ} 5653$ 
In (9) we can define independent random variables $V, E_{k}, k=0,1, \ldots$, $N$ and $X$ where $V$ has density $A$, the $E_{k}$ 's are exponential with parameter $(\mu+\beta) \theta^{k}, N$ is geometric with $P(N=n)=\left(1-\frac{\beta}{\mu+\beta}\right)\left(\frac{\beta}{\mu+\beta}\right)^{n}$ and $X$ takes the values 0 or 1 with probabilities $(1-\delta / \mu)$ and $\delta / \mu$. By inspection, $\widehat{f}$ is the Laplace transform $E \exp (-s W)$ of the random variable

$$
W=(1-X) \sum_{n=0}^{N} E_{n}+X\left(\frac{V}{\theta^{N}}+\sum_{n=0}^{N} E_{n}\right) .
$$

We have therefore established the existence and uniqueness of a density solution to (9).

We can calculate the inverse of the Laplace transform given at (13). First remark that the density giving the transform

$$
\prod_{k=0}^{n} \frac{\mu+\beta}{\mu+\beta+s / \theta^{k}}
$$

can be written as $g_{n}(z)=\sum_{k=0}^{n} d_{k, n} \exp \left(-(\mu+\beta) \theta^{k} z\right)$ where

$$
d_{k, n}=(\mu+\beta) b_{k} \prod_{i=1}^{n-k} \frac{1}{1-\theta^{-i}} \text { with } \prod_{i=1}^{0} \frac{1}{1-\theta^{-i}}=1
$$

This holds because the transform of $\sum_{k=0}^{n} d_{k, n} \exp \left(-(\mu+\beta) \theta^{k} z\right)$ is

$$
\sum_{k=0}^{n} d_{k, n}\left(\frac{1}{(\mu+\beta) \theta^{k}+s}\right)
$$


where $d_{k, n}$ are found by using

$$
\begin{aligned}
d_{k, n} & =(\mu+\beta) \theta^{k} \prod_{i=0}^{k-1}\left(\frac{(\mu+\beta) \theta^{i}}{(\mu+\beta) \theta^{i}-(\mu+\beta) \theta^{k}}\right) \prod_{i=k+1}^{n}\left(\frac{(\mu+\beta) \theta^{i}}{(\mu+\beta) \theta^{i}-(\mu+\beta) \theta^{k}}\right) \\
& =(\mu+\beta) \theta^{k} \prod_{i=0}^{k-1}\left(\frac{1}{1-\theta^{k-i}}\right) \prod_{i=k+1}^{n}\left(\frac{\theta^{i-k}}{\theta^{i-k}-1}\right) \\
& =(\mu+\beta) \prod_{i=1}^{k}\left(\frac{\theta}{1-\theta^{i}}\right) \prod_{i=1}^{n-k}\left(\frac{\theta^{i}}{\theta^{i}-1}\right) .
\end{aligned}
$$

Let $\aleph(\cdot)$ denote the measure $\left(1-\frac{\delta}{\mu}\right) \delta_{0}(d x)+\frac{\delta}{\mu} A(x) d x$. The density of the variable $W$ at $y$, conditioned on $N=n$, is given by

$$
\begin{aligned}
\int_{0}^{\theta^{n} y} g_{n}\left(y-\frac{x}{\theta^{n}}\right) \aleph(d x) & =\int_{0}^{\theta^{n} y} \sum_{k=0}^{n} d_{k, n} e^{-(\mu+\beta) \theta^{k}\left(y-\frac{x}{\theta^{n}}\right)} \aleph(d x) \\
& =\sum_{k=0}^{n} d_{k, n} e^{-(\mu+\beta) \theta^{k} y} \int_{0}^{\theta^{n} y} e^{(\mu+\beta) x / \theta^{n-k}} \aleph(d x) .
\end{aligned}
$$

Note that the above integral exists by the assumption (7), i.e., $\int_{0}^{\infty} e^{(\mu+\beta) z} A(z) d z<\infty$. Now summing over $n$ we have the density for $W$ at $y$ :

$$
\begin{aligned}
& \sum_{n \geq 0}\left(1-\frac{\beta}{\mu+\beta}\right)\left(\frac{\beta}{\mu+\beta}\right)^{n} \sum_{k=0}^{n} d_{k, n} e^{-(\mu+\beta) \theta^{k} y} \int_{0}^{\theta^{n} y} e^{(\mu+\beta) x / \theta^{n-k}} \aleph(d x) \\
= & \sum_{k \geq 0}(\mu+\beta) b_{k}\left(1-\frac{\beta}{\mu+\beta}\right)\left(\frac{\beta}{\mu+\beta}\right)^{k} e^{-(\mu+\beta) \theta^{k} y} \\
& \cdot \sum_{n \geq k}\left(\frac{\beta}{\mu+\beta}\right)^{n-k}\left(\prod_{i=1}^{n-k} \frac{1}{1-\theta^{-i}}\right) \int_{0}^{\theta^{n} y} e^{(\mu+\beta) x / \theta^{n-k}} \aleph(d x) \\
= & \sum_{k \geq 0}(\mu+\beta) b_{k}\left(1-\frac{\beta}{\mu+\beta}\right)\left(\frac{\beta}{\mu+\beta}\right)^{k} e^{-(\mu+\beta) \theta^{k} y} \\
& \cdot \sum_{m \geq 0}\left(\frac{\beta}{\mu+\beta}\right)^{m}\left(\prod_{i=1}^{m} \frac{1}{1-\theta^{-i}}\right) \int_{0}^{\theta^{k+m} y} e^{(\mu+\beta) x / \theta^{m}} \aleph(d x) .
\end{aligned}
$$

$\mathrm{RR} \mathrm{n}^{\circ} 5653$ 


\section{The Rate-Dependent Case $(\gamma>1)$}

\subsection{The persistent case $(\mu=\delta=0)$}

Define $h$ to be a density solution of (11) with $\beta$ replaced by $\beta / \gamma$ so

$$
h(z)=\psi \frac{\beta}{\gamma} \sum_{n \geq 0} b_{n} e^{-\left(\frac{\beta}{\gamma} \theta^{n}\right) z} .
$$

Next, for all positive constants $C, f(z)=C^{-1} h\left(z^{\gamma}\right)$ satisfies

$$
\begin{aligned}
\frac{d f(z)}{d z} & =C^{-1} \frac{d h\left(z^{\gamma}\right)}{d z} \\
& =C^{-1} \gamma z^{\gamma-1} \frac{\beta}{\gamma}\left(\theta h\left(\theta z^{\gamma}\right)-h\left(z^{\gamma}\right)\right) \\
& =C^{-1} \beta z^{\gamma-1}\left(\rho^{\gamma} h\left((\rho z)^{\gamma}\right)-h\left(z^{\gamma}\right)\right) \quad \text { where } \rho^{\gamma}=\theta \\
& =\beta z^{\gamma-1}\left(\rho^{\gamma} f(\rho z)-f(z)\right) .
\end{aligned}
$$

So if one takes $C$ such that $\int_{0}^{\infty} f(z) d z=1$, then $f$ is a density which satisfies (4).

We can understand this transformation as a change of measure. Let $I$ be a random variable with distribution $h$ given by (14). We note that for any bounded function $\phi$,

$$
\begin{aligned}
\frac{1}{C} E\left[\frac{\phi\left(I^{1 / \gamma}\right)}{\gamma I^{1-1 / \gamma}}\right] & =\frac{1}{C} \int_{0}^{\infty} \phi\left(z^{1 / \gamma}\right) \frac{1}{\gamma z^{1-1 / \gamma}} h(z) d z \\
& =\frac{1}{C} \int_{0}^{\infty} \phi(w) h\left(w^{\gamma}\right) d w \\
& =\int_{0}^{\infty} \phi(w) f(w) d w \\
& =E[\phi(J)]
\end{aligned}
$$

where $J$ is a random variable with a density given by $f(z)$. 
We can use this change of measure to compute the normalizing constant:

$$
\begin{aligned}
C & =E\left[\frac{1}{\gamma I^{1-1 / \gamma}}\right] \\
& =\frac{1}{\gamma} \int_{0}^{\infty} \frac{1}{z^{1-1 / \gamma}} h(z) d z \\
& =\frac{\psi \beta}{\gamma^{2}} \sum_{n \geq 0} b_{n} \int_{0}^{\infty} \frac{1}{z^{1-1 / \gamma}} \exp \left(-\frac{\beta}{\gamma} \theta^{n} z\right) d z \\
& =\frac{\psi}{\gamma} \sum_{n \geq 0} b_{n} \frac{1}{\theta^{n}}\left(\frac{\gamma}{\beta \theta^{n}}\right)^{1 / \gamma-1} \int_{0}^{\infty} v^{1 / \gamma-1} \exp (-v) d v \\
& =\frac{\psi}{\gamma}\left(\frac{\gamma}{\beta}\right)^{1 / \gamma-1} \Gamma\left(\frac{1}{\gamma}\right) \sum_{n \geq 0} b_{n}\left(\frac{1}{\theta^{1 / \gamma}}\right)^{n} \\
& =\frac{\psi}{\gamma}\left(\frac{\beta}{\gamma}\right)^{1-1 / \gamma} \Gamma\left(\frac{1}{\gamma}\right) \prod_{k \geq 0}\left(1-\theta^{-k-1 / \gamma}\right) .
\end{aligned}
$$

Hence (5) is a density solution to (4). By a similar argument, taking $\phi(I)=I$, we get that the mean value of $f$ is

$$
\begin{aligned}
\int_{0}^{\infty} z f(z) d z & =\frac{E\left[I^{2 / \gamma-1}\right]}{C \gamma} \\
& =\frac{1}{C \gamma} \int_{0}^{\infty} z^{2 / \gamma-1} h(z) d z \\
& =\frac{\psi \beta}{C \gamma^{2}} \sum_{n \geq 0} b_{n} \int_{0}^{\infty} z^{2 / \gamma-1} \exp \left(-\frac{\beta}{\gamma} \theta^{n} z\right) d z \\
& =\frac{\psi}{C \gamma} \sum_{n \geq 0} b_{n} \frac{1}{\theta^{n}}\left(\frac{\gamma}{\beta \theta^{n}}\right)^{2 / \gamma-1} \int_{0}^{\infty} v^{2 / \gamma-1} \exp (-v) d v \\
& =\frac{\psi}{C \gamma}\left(\frac{\gamma}{\beta}\right)^{2 / \gamma-1} \Gamma\left(\frac{2}{\gamma}\right) \sum_{n \geq 0} b_{n}\left(\frac{1}{\theta^{2 / \gamma}}\right)^{n} \\
& =\frac{\psi}{C \gamma}\left(\frac{\beta}{\gamma}\right)^{1-2 / \gamma} \Gamma\left(\frac{2}{\gamma}\right) \prod_{k \geq 0}\left(1-\theta^{-k-2 / \gamma}\right)
\end{aligned}
$$

$\mathrm{RR} \mathrm{n}^{\circ} 5653$ 
This proves (6).

We prove now that the unique density solution of (4) is (5).

Suppose there is a density solution $f$ of (4). Define $h$ via $f(z)=C^{-1} h\left(z^{\gamma}\right)$ to be a solution of (11). Since $f$ is integrable this means $\infty>C^{-1} \int_{0}^{\infty} h\left(z^{\gamma}\right) d z=$ $\frac{C^{-1}}{\gamma} \int_{0}^{\infty} s^{\frac{1}{\gamma}-1} h(s) d s=\frac{C^{-1}}{\gamma} H\left(\frac{1}{\gamma}\right)$, where $H(u)=\int_{0}^{\infty} x^{u-1} h(s) d s$ is the Mellin transform of $h$. Multiplying both sides of (11) by $z^{u}$ and integrating with respect to $z$ on the positive half line we get

$$
u H(u)=\beta H(u+1)\left(1-\theta^{-u}\right) .
$$

Since $H(1 / \gamma)<\infty$ we see $H(u)<\infty$ for all $u$ by iteration. This means $\int_{0}^{\infty} h(z) d z=H(1)<\infty$ so $h$ can be normalized to a density. However we have shown that there is only one density satisfying (11) so $h$ is in fact (11). We conclude that there is only one density solution to (4) because two density solutions in the rate-dependent case would give two density solutions in the rate-independent case using the transformation. We have just proved this is impossible and this proves uniqueness and completes the proof of Theorem 1.

\subsection{The non-persistent case $(\mu>\delta \geq 0)$}

Let $A$ be some density. Then $\frac{A\left(z^{1 / \gamma}\right)}{\gamma z^{1-1 / \gamma}}$ is a density too. Let $h$ be the density solution of

$$
\frac{d h}{d z}(z)=\frac{1}{\gamma}\left(\left(\frac{A\left(z^{1 / \gamma}\right)}{\gamma z^{1-1 / \gamma}}\right)-\mu h(z)+\beta(\theta h(\theta z)-h(z))\right) .
$$

We assume $h(0)=\mu-\delta \geq 0$.

Let $f(z)=C^{-1} h\left(z^{\gamma}\right)$, where $C$ normalizes $f$ to a density, and $\rho^{\gamma}=\theta$. Then we have

$$
\begin{aligned}
\frac{d f(z)}{d z} & =C^{-1} \delta \gamma^{-1} A(z)-\mu z^{\gamma-1} f(z)+\beta z^{\gamma-1}\left(\rho^{\gamma} f(\rho z)-f(z)\right) \\
& =\delta T(f) A(z)-\mu z^{\gamma-1} f(z)+\beta z^{\gamma-1}\left(\rho^{\gamma} f(\rho z)-f(z)\right),
\end{aligned}
$$

where we used the fact that

$$
T(f)=\int_{0}^{\infty} z^{\gamma-1} C^{-1} h\left(z^{\gamma}\right) d z=\frac{1}{C \gamma} .
$$


Then when denoting $h(z)$ the unique solution of (15) which is a density, we see that $f(z)$ is a positive solution of (3). Hence if $f(z)$ is integrable, it is a density for some normalizing constant $C$.

We can use the same change of measure as in the persistent case. Let $I$ be the random variable with distribution $h$ defined above. We have

$$
\begin{aligned}
C & =E\left[\frac{1}{\gamma I^{1-1 / \gamma}}\right] \\
& =\frac{1}{\gamma} \int_{0}^{\infty} y^{1 / \gamma-1} h(y) d y \\
& =\frac{\mu}{\gamma^{2}} \sum_{k \geq 0} b_{k}\left(\frac{\beta}{\mu+\beta}\right)^{k} \cdot \sum_{m \geq 0} c_{m} \int_{0}^{\infty} y^{1 / \gamma-1} e^{-\frac{\mu+\beta}{\gamma} \theta^{k} y} \int_{0}^{\theta^{k+m} y} e^{\frac{(\mu+\beta) x}{\gamma \theta^{m}}} \aleph(d x) d y
\end{aligned}
$$

with

$$
\aleph(d x)=\frac{\delta}{\mu}\left(\frac{A\left(x^{1 / \gamma}\right)}{\gamma x^{1-1 / \gamma}}\right) d x+\left(1-\frac{\delta}{\mu}\right) \delta_{0}(d x) .
$$

To simplify,

$$
\begin{aligned}
& \int_{0}^{\infty} y^{1 / \gamma-1} e^{-\frac{\mu+\beta}{\gamma} \theta^{k} y} \int_{0}^{\theta^{k+m} y} e^{\frac{(\mu+\beta) x}{\gamma \theta^{m}}} \aleph(d x) d y \\
= & \int_{0}^{\infty} e^{\frac{(\mu+\beta) x}{\gamma^{m}}} \aleph(d x) \int_{x / \theta^{(k+m)}}^{\infty} y^{1 / \gamma-1} e^{-\frac{\mu+\beta}{\gamma} \theta^{k} y} d y \\
= & \frac{1}{\theta^{k / \gamma}} \frac{\delta}{\mu} \int_{0}^{\infty} e^{\frac{(\mu+\beta) x}{\gamma \theta^{m}}}\left(\frac{A\left(x^{1 / \gamma}\right)}{\gamma x^{1-1 / \gamma}}\right) \int_{x / \theta^{m}}^{\infty} B_{1}(u) d u d x \\
& +\frac{1}{\theta^{k / \gamma}}\left(1-\frac{\delta}{\mu}\right)\left(\frac{\mu+\beta}{\gamma}\right)^{-1 / \gamma} \Gamma\left(\frac{1}{\gamma}\right) \\
= & \frac{1}{\theta^{k / \gamma}} \frac{\delta}{\mu} \int_{0}^{\infty} e^{\frac{(\mu+\beta) x^{\gamma}}{\gamma \theta^{m}}} A(x) \int_{x^{\gamma / \theta^{m}}}^{\infty} B_{1}(u) d u d x \\
& +\frac{1}{\theta^{k / \gamma}}\left(1-\frac{\delta}{\mu}\right)\left(\frac{\mu+\beta}{\gamma}\right)^{-1 / \gamma} \Gamma\left(\frac{1}{\gamma}\right) .
\end{aligned}
$$

$\mathrm{RR} \mathrm{n}^{\circ} 5653$ 
Substituting, we get

$$
\begin{aligned}
C & =G_{1} \frac{\mu}{\gamma^{2}} \sum_{k \geq 0} b_{k}\left(\frac{\beta}{\mu+\beta}\right)^{k} \frac{1}{\theta^{k / \gamma}} \\
& =G_{1} \frac{\mu}{\gamma^{2}} \prod_{m \geq 0}\left(1-\frac{\beta}{\mu+\beta} \theta^{-m-1 / \gamma}\right)
\end{aligned}
$$

with

$$
\begin{aligned}
G_{1}= & \sum_{m \geq 0} c_{m}\left(\frac{\delta}{\mu} \int_{0}^{\infty} \bar{A}_{m}(x) \int_{x^{\gamma} / \theta^{m}}^{\infty} B_{1}(u) d u d x\right. \\
& \left.+\left(1-\frac{\delta}{\mu}\right)\left(\frac{\mu+\beta}{\gamma}\right)^{-1 / \gamma} \Gamma\left(\frac{1}{\gamma}\right)\right)
\end{aligned}
$$

The last series is convergent because of the assumption (7), i.e., $\int_{0}^{\infty} e^{\frac{(\mu+\beta)}{\gamma} z^{\gamma}} A(z) d z<$ $\infty$ and the fact that

$$
\int_{x / \theta^{m}}^{\infty} \frac{1}{u^{1-1 / \gamma}} e^{-\frac{(\mu+\beta)}{\gamma} u} d u \leq\left(\frac{\gamma}{\beta+\gamma}\right)^{1 / \gamma} \Gamma\left(\frac{1}{\gamma}\right) .
$$

The mean value of $f$ is

$$
\begin{aligned}
\int_{0}^{\infty} z f(z) d z \\
=\frac{E\left[I^{2 / \gamma-1}\right]}{C \gamma} \\
=\frac{1}{C \gamma} \int_{0}^{\infty} y^{2 / \gamma-1} h(y) d y \\
=\frac{\mu}{C \gamma^{2}} \sum_{k \geq 0} b_{k}\left(\frac{\beta}{\mu+\beta}\right)^{k}\left(\sum_{m \geq 0} c_{m} \int_{0}^{\infty} y^{2 / \gamma-1} e^{-\frac{\mu+\beta}{\gamma} \theta^{k} y} \cdot \int_{0}^{\theta^{k+m} y} e^{\frac{(\mu+\beta) x}{\gamma \theta^{m}}} \aleph(d x) d y\right) .
\end{aligned}
$$

We have

$$
\begin{aligned}
& \int_{0}^{\infty} y^{2 / \gamma-1} e^{-\frac{\mu+\beta}{\gamma} \theta^{k} y} \int_{0}^{\theta^{k+m} y} e^{\frac{(\mu+\beta) x}{\gamma \theta^{m}}} \aleph(d x) d y \\
= & \frac{1}{\theta^{2 k / \gamma}} \int_{0}^{\infty} e^{\frac{(\mu+\beta) x}{\gamma \theta^{m}}} \aleph(d x) \int_{x / \theta^{m}}^{\infty} u^{2 / \gamma-1} e^{-\frac{\mu+\beta}{\gamma} u} d u .
\end{aligned}
$$


Substituting we get

$$
\begin{aligned}
\int_{0}^{\infty} z f(z) d z & =G_{2} \frac{\mu}{C \gamma^{2}} \sum_{k \geq 0} b_{k}\left(\frac{\beta}{\mu+\beta}\right)^{k} \frac{1}{\theta^{2 k / \gamma}} \\
& =G_{2} \frac{\mu}{C \gamma^{2}} \prod_{m \geq 0}\left(1-\frac{\beta}{\mu+\beta} \theta^{-m-2 / \gamma}\right)
\end{aligned}
$$

with

$$
\begin{aligned}
G_{2}= & \sum_{m \geq 0} c_{m}\left(\frac{\delta}{\mu} \int_{0}^{\infty} \bar{A}_{m}(x) \int_{x^{\gamma} / \theta^{m}}^{\infty} u^{2 / \gamma-1} e^{-\frac{\mu+\beta}{\gamma} u} d u d x\right. \\
& \left.+\left(1-\frac{\delta}{\mu}\right)\left(\frac{\mu+\beta}{\gamma}\right)^{-2 / \gamma} \Gamma\left(\frac{2}{\gamma}\right)\right) .
\end{aligned}
$$

The arguments showing that the last series is convergent are similar to those for $G_{1}$ above.

Moreover, by the same argument as above, we can show that $H(1 / \gamma)$ is finite where $H$ the Mellin transform of $h$ and by iteration $H(u)$ is analytic for all $u$. We again conclude there is a unique solution to the non-persistent rate-dependent case.

Remark 1 We have $f(0)=T(f)(\mu-\delta)$. When taking $z=0$ in (8), one gets that

$$
f(0)=T(f)(\mu-\delta)\left(\sum_{m \geq 0} c_{m}\right)\left(\sum_{k \geq 0}\left(\frac{\beta}{\beta+\mu}\right)^{k} b_{k}\right) .
$$

This leads to the following interesting identity (which can be checked directly): for all $0 \leq x<1$,

$$
\left(\sum_{m \geq 0} x^{m} \prod_{i=1}^{m} \frac{1}{1-\theta^{-i}}\right)\left(\sum_{k \geq 0} x^{k} \prod_{p=1}^{k} \frac{\theta}{1-\theta^{p}}\right)=1 .
$$

$\mathrm{RR} \mathrm{n}^{\circ} 5653$ 


\section{The Transient Case}

Consider the following rate-independent partial differential equation:

$$
\alpha \frac{\partial}{\partial t} f(t, z)+\frac{\partial}{\partial z} f(t, z)=\beta(\theta f(t, \theta z)-f(t, z)), \quad z, t \geq 0
$$

where we suppose that $f(0, z)$ is integrable. Defining

$$
\widehat{f}(u, s)=\int_{0}^{\infty} \int_{0}^{\infty} e^{-u t-s z} f(t, z) d t d z
$$

we get that

$$
(\beta+\alpha u+s) \widehat{f}(u, s)=\beta \widehat{f}\left(u, \frac{s}{\theta}\right)+\alpha \widehat{f}_{0}(s)+\widehat{f}_{1}(u)
$$

with

$$
\widehat{f}_{0}(s)=\int_{0}^{\infty} e^{-s z} f(0, z) d z \quad \text { and } \quad \widehat{f}_{1}(u)=\int_{0}^{\infty} e^{-u t} f(t, 0) d t .
$$

Recall we are seeking a solution such that $f(t, z)$ is a density in $z$ for each $t$. Hence $\widehat{f}(u, 0)=1 / u$ and $\widehat{f}_{0}(0)=1$. Substitute $s=0$ into (17) so

$$
(\beta+\alpha u) / u=\beta / u+\alpha+\widehat{f}_{1}(u)
$$

and it follows that $\widehat{f}_{1}(u)=0$.

Hence

$$
\widehat{f}(u, s)=\sum_{k=-1}^{\infty}\left(\prod_{l=0}^{k} \frac{\beta}{\beta+\alpha u+\frac{s}{\theta^{l}}}\right)\left(\frac{\alpha}{\beta+\alpha u+\frac{s}{\theta^{k+1}}}\right) \widehat{f}_{0}\left(\frac{s}{\theta^{k+1}}\right) .
$$

The function

$$
\left(\prod_{l=0}^{k} \frac{\beta / \alpha}{\beta / \alpha+u+\frac{s}{\alpha \theta^{l}}}\right)\left(\frac{\beta / \alpha}{\beta / \alpha+u+\frac{s}{\alpha \theta^{k+1}}}\right) \cdot \widehat{f}_{0}\left(\frac{s}{\theta^{k+1}}\right)
$$

is the joint Laplace transform $E\left(e^{-u Y-s W}\right)$ with

$$
W=W(0) / \theta^{k+1}+\frac{1}{\alpha}\left(\sum_{l=0}^{k+1} \frac{E_{l}}{\theta^{l}}\right) \quad \text { and } \quad Y=\sum_{l=0}^{k+1} E_{l},
$$

where $E_{l}$ are i.i.d. exponential random variables with parameter $\beta / \alpha$ and $W(0)$ is an independent random variable distributed like $f(0, z)$. 


\section{References}

[1] O. Diekmann, "The Cell Size Distribution and Semigroups of Linear Operators." Lecture notes in biomathematics: The dynamics of physiologically structured populations; Metz, J.A.J ed. Springer, 1986.

[2] R. Stevens and G. Wright, "TCP illustrated", Addison Wesley, 2001.

[3] F. Baccelli, D. R. McDonald, and J. Reynier, "A Mean-Field Model for Multiple TCP Connections through a Buffer Implementing RED", Performance Evaluation, vol. 49, pp. 77-97, 2002.

[4] F. Baccelli and D. McDonald, "A Square Root Formula for the Rate of Non Persistent HTTP Flows". INRIA Report Number 5301, 2004.

[5] F. Baccelli, K. B. Kim, and D. D. Vleeschauwer, "Analysis of the Competition Between Wired, DSL and Wireless Users in an Access Network," IEEE Infocom 05, Miami, 2005.

[6] B. Perthame and L. Ryzhik, "Exponential Decay for the Fragmentation or Cell-Division Equation," J. Differential Equations, vol. 210, no. 1, pp. 155$177,2005$.

$\mathrm{RR} \mathrm{n}^{\circ} 5653$ 


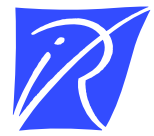

Unité de recherche INRIA Rocquencourt

Domaine de Voluceau - Rocquencourt - BP 105 - 78153 Le Chesnay Cedex (France)

Unité de recherche INRIA Futurs : Parc Club Orsay Université - ZAC des Vignes

4, rue Jacques Monod - 91893 ORSAY Cedex (France)

Unité de recherche INRIA Lorraine : LORIA, Technopôle de Nancy-Brabois - Campus scientifique

615, rue du Jardin Botanique - BP 101 - 54602 Villers-lès-Nancy Cedex (France)

Unité de recherche INRIA Rennes : IRISA, Campus universitaire de Beaulieu - 35042 Rennes Cedex (France)

Unité de recherche INRIA Rhône-Alpes : 655, avenue de l'Europe - 38334 Montbonnot Saint-Ismier (France)

Unité de recherche INRIA Sophia Antipolis : 2004, route des Lucioles - BP 93 - 06902 Sophia Antipolis Cedex (France)

INRIA - Domaine de Voluceau - Rocquencourt, BP 105 - 78153 Le Chesnay Cedex (France)

http://www.inria.fr

ISSN 0249-6399 